\title{
Fast damping in mismatched high intensity beam transportation
}

\author{
V. Variale \\ INFN Sezione di Bari, Via Amendola 173, 70126-Bari, Italy
}

(Received 18 December 2000; published 8 August 2001)

\begin{abstract}
A very fast damping of beam envelope oscillation amplitudes was recently observed in simulations of high intensity beam transport, through periodic FODO cells, in mismatched conditions [V. Variale, Nuovo Cimento Soc. Ital. Fis. 112A, 1571-1582 (1999) and T. Clauser et al., in Proceedings of the Particle Accelerator Conference, New York, 1999 (IEEE, Piscataway, NJ, 1999), p. 1779]. A Landau damping mechanism was proposed at the origin of observed effect. In this paper, to further investigate the source of this fast damping, extensive simulations have been carried out. The results presented here support the interpretation of the mechanism at the origin of the fast damping as a Landau damping effect.
\end{abstract}

DOI: 10.1103/PhysRevSTAB.4.084201

PACS numbers: 29.27.Bd, 29.27.Eg, 41.85.-p

\section{INTRODUCTION}

High intensity charged-particle beams can develop extended low-density halos [1]. The existence of halos can have serious consequences in particle accelerators. If halo particles are lost in the accelerator they may induce radioactivity. For the next generation of high intensity proton linacs, it is necessary to obtain a more quantitative understanding of the physics of the halo. Multiparticle simulation studies of high intensity beams transported in linear focusing channels have provided some useful physical insights into the dynamics of high-current beams. For a nonequilibrium beam injected with the correctly matched rms size, the initial distribution relaxes with accompanying emittance growth over a time of only about one-quarter plasma period to a quasiequilibrium state with an approximately uniform central core in real space and with an edge that falls off over a distance approximately equal to the Debye length [2]. Furthermore, numerical simulations of rms-mismatched beams have shown that the beam envelope oscillations, due to mismatching, induce a halo on the beam that could cause radioactive activation on the beam surrounding [3]. Recently, multiparticle code simulations have shown that beam envelope oscillations, caused by mismatching with the periodic transport channel, can damp on a very fast time scale [4,5]. In these references, a continuous beam was transported through hundreds of FODO periodic cells. Furthermore, a comparison of the simulation results obtained either with a multiparticle code (PARMT) or a particle core model (PCM) led to the following conclusions. PCM calculations seem unsuitable to study test particles taken near the beam core region in the case of high beam current. The discrepancies found between the multiparticle code and PCM results were mainly due to the following reasons: In PARMT simulations, the breathing mode oscillations, due to the mismatch of the input beam with the periodic cells, damped very quickly when the beam space charge was strong enough. In PCM calculations, however, a constant amplitude oscillation of the beam envelope was considered.

In this paper, an attempt to better understand the mechanism causing the fast damping of the beam breathing mode oscillations observed in Ref. [4] will be presented, with the assumption that this effect can be ascribed to a Landau mechanism of stabilization.

The Landau damping mechanism applies to a set of oscillators, with a frequency distribution $f\left(\omega_{\beta}\right)$, coupled to a harmonic excitation of frequency $\omega$. The response of the set to the excitation can be seen by the center-of-charge velocity of the set [6]

$$
\langle\dot{y}\rangle=\hat{G} e^{i \omega t}\left[\pi f(\omega)-i P \int \frac{f\left(\omega_{\beta}\right)}{\omega_{\beta}-\omega} d \omega_{\beta}\right]
$$

where $P$ is the principal value integral and $\hat{G}$ is the amplitude of the harmonic excitation. This response has a real part for which the velocity and the excitations are in phase and an imaginary part where they are out of phase, indicating no energy exchange. The real term indicates that the beam absorbs energy from the exciting signal. The energy absorption can lead to the damping of the coherent oscillation often called Landau damping. From Eq. (1) one can notice that the real term is proportional to the density of the frequency distribution $f\left(\omega_{\beta}\right)$ in the region of the excitation frequency $\omega$. Therefore, a condition to obtain the Landau damping is that the exciting frequency lies inside the incoherent frequency distribution. Furthermore, Eq. (1) is correct only if the excitation lasts for a long time. How long this time has to be depends on the shape of the frequency distribution $f\left(\omega_{\beta}\right)$ considered. If this distribution does not change significantly over a frequency range of $\Delta \omega_{\beta}$, around $\omega$, it is sufficient to excite for a time $\tau \gg 1 / \Delta \omega_{\beta}$. In this case, all of the oscillations with frequency inside $\Delta \omega_{\beta}$, in time $t$, are still responding positively to the exciting force. Then, to have a large period of 
interaction between the exciting force and the oscillations, we need a large $\Delta \omega_{\beta}$.

In conclusion, the Landau damping mechanism applies only if we have oscillators with a sufficiently large frequency spectrum covering the exciting frequency. Furthermore, the damping strength is proportional to the value of the frequency distribution function $f\left(\omega_{\beta}\right)$ taken at the excitation frequency $\omega$.

\section{SIMULATIONS}

The multiparticle code PARMT is a Monte Carlo program that can transport an ion beam through a system of optics elements by using the matrix method [7]. In order to simulate a very high number of particles, PARMT employs a fast Poisson solver technique to compute the space charge electric field of the ion beam. The fast damping effect of the rms envelope oscillations, for a continuous beam transported through FODO cells, in mismatched conditions, was shown in Ref. [4]. The parameter simulations used in Ref. [4] were FODO cell periods of length $L=80 \mathrm{~cm}$, transverse rms emittances $\varepsilon_{x}=\varepsilon_{y}=0.25 \times 10^{-6} \mathrm{~m} \mathrm{rad}$, single particle phase advance $\sigma_{0}=60.7^{\circ}$, and a beam current of $95 \mathrm{~mA}$ with a relativistic parameter $\beta=0.145$. Moreover, an odd or even mismatching of about $10 \%$ was added on the initial beam size. The fast oscillation damping of the beam odd breathing mode, found in Ref. [4], is represented in Fig. 1(a). The odd mode is embodied by the black line while the magenta line refers to the even mode. It is interesting to notice that, in Fig. 1(b), the related "total emittance variation" $e_{t}$, defined as $e_{t}=\left(\varepsilon_{x}^{2} / \varepsilon_{x 0}^{2}+\right.$ $\left.\varepsilon_{y}^{2} / \varepsilon_{y 0}^{2}\right)^{1 / 2}$, increases while the rms envelope amplitudes decrease. As already argued in Ref. [4], the damping effect, shown in Fig. 1, can be ascribed to the Landau mechanism of stabilization briefly described above. This mechanism is already used in accelerator physics to explain the Keil-Schnell stability criterion. The Landau damping can be considered a stabilizing effect connected with the frequency spread [8].

To interpret the behavior of Fig. 1 in the framework of the Landau damping mechanism, some preliminary considerations on the studied system have to be made. The beam particles, in the reference particle system, can be considered as a set of oscillators that oscillate with their betatron frequency because of the focusing forces. A betatron frequency spread will be present, e.g., due to cromaticity and momentum spread. While, in general, this frequency spread tends to be very small when space charge dominated beams ${ }^{1}$ are considered, an enhancement can be observed. In fact, the space charge force induces a beta-

\footnotetext{
${ }^{1}$ A space charge dominated beam has $K a^{2} / 4 \varepsilon^{2} \gg 1$ [9], where $K=q I /\left(2 \pi \varepsilon_{0} \gamma^{3} \beta^{3} m c^{3}\right)$ is the generalized perveance, $a$ is the rms transverse beam size, and $\varepsilon$ is the transverse emittance.
}
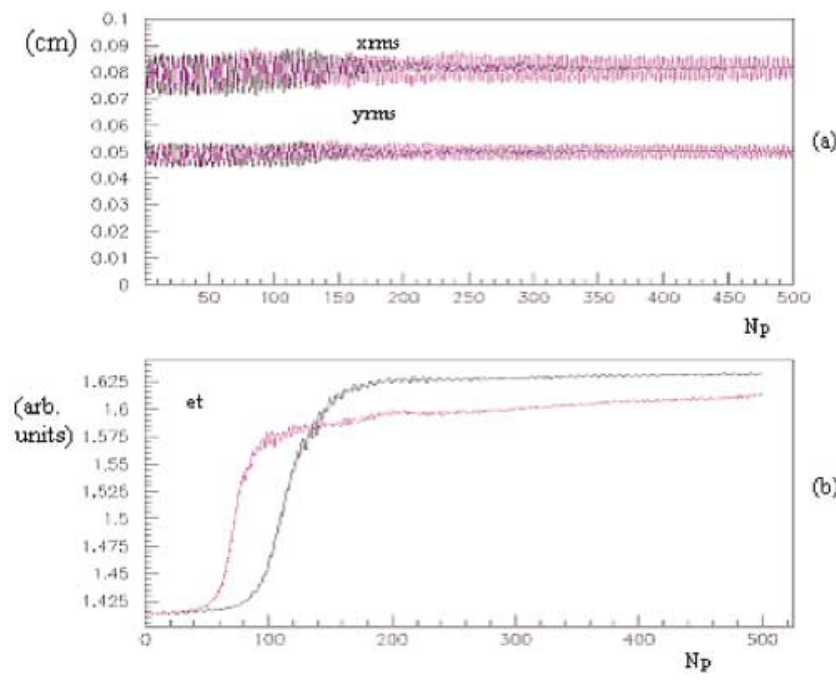

FIG. 1. (Color) (a) rms envelope oscillations with initial $\mathrm{K}-\mathrm{V}$ distribution for $\sigma / \sigma_{0}=0.55$ and (b) the total emittance variation $e_{t}$ vs the periodic cell number $N p$. The black line refers to the odd mode and the magenta line to the even mode.

tron tune shift on the beam particles. In general, it is an incoherent tune shift causing an increase of the betatron frequency spread. However, in the case of a K-V distribution, the space charge force is linear due to uniform spatial distribution. As a consequence, the space charge tune shift is uniform; that is, all the particles will oscillate with the same shifted betatron frequency. No increase in betatron spectrum should be observed as long as this
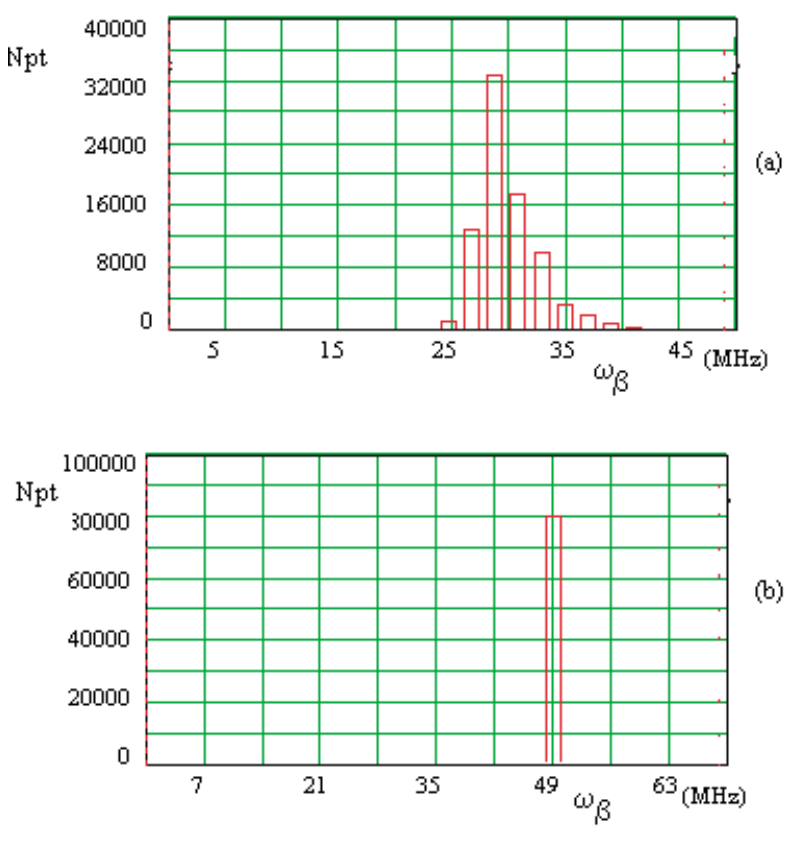

FIG. 2. (Color) Beam betatron frequency distribution: (a) case for $\sigma / \sigma_{0}=0.55$ and (b) case for $\sigma / \sigma_{0}=0.83$. Npt is the particle number. 
distribution is sustained. This means that Landau damping mechanism cannot be applied for the K-V distribution. This should also be the case for the simulation results of Fig. 1, where an initial K-V distribution was used. However, for an initial K-V distribution, it is known (see, for example, Ref. [10]) that, if the space charge force is strong enough, it can rearrange the internal charges toward a new distribution that is uniform in the central part and with a tail length $\lambda_{d}$ (Debye length) on the edge. In these conditions, the space charge field is no longer linear and the betatron spectrum is no longer a Dirac delta. In the simulation results shown in Fig. 1, a space charge tune depression $\sigma / \sigma_{0}$ of 0.55 (corresponding in our case to $I=95 \mathrm{~mA}$ ) was used. This value gives a space charge capable of rearranging the charges of the initial $\mathrm{K}-\mathrm{V}$ distribution. This is evident in Fig. 2(a), which shows the betatron frequency distribution of the beam used in Fig. 1. For comparison, in Fig. 2(b) the betatron frequency distribution of a beam with $\sigma / \sigma_{0}=0.83$ (corresponding to $I=24 \mathrm{~mA}$ ) is also shown. In the latter case, the space charge is not strong enough to modify the K-V distribution and the betatron spectrum remains a Dirac delta.

\section{ENVELOPE OSCILLATIONS}

A beam in a periodic focusing channel is matched if the beam envelope repeats itself after each focusing period. In this case, the graphics of the envelope value versus the period number $N p$ is a line of a constant value. In the case of mismatching, the envelope value, at the exit of the focusing cell, is no longer constant and the graphics show oscillations, as in Fig. 1(a).

In Ref. [11], the theory for the envelope oscillations of mismatched beams is presented. It shows that the envelope behavior can be described by the equations

$$
\begin{aligned}
& z_{1}^{\prime \prime}+k_{1}^{2} z_{1}=0 \rightarrow k_{1}^{2}=\left(\sigma_{0}^{2}+3 \sigma^{2}\right) / S^{2} \text { (odd), } \\
& z_{2}^{\prime \prime}+k_{2}^{2} z_{2}=0 \rightarrow k_{2}^{2}=\left(2 \sigma_{0}^{2}+2 \sigma^{2}\right) / S^{2} \text { (even), }
\end{aligned}
$$

where $S$ indicates the focusing periodic cell length, $z_{1}=$ $x(s)-y(s)$ and $z_{2}=x(s)+y(s)$ are the odd and even modes of the rms envelopes, $x(s)$ and $y(s)$ are the deviations from the transverse beam envelopes, $X(s)$ and $Y(s)$, and $s$ is the longitudinal position along the periodic cell.

Notice that any arbitrary envelope oscillation mode can be expressed as a combination of these two fundamental modes (odd and even). The spatial frequencies $k_{1}$ and $k_{2}$ can be expressed as phase advances $\Phi_{1}=k_{1} S$ and $\Phi_{2}=k_{2} S$. The phase advances obtained by this theory have been found in very good agreement with those given by the simulation results of the multiparticle code PARMT.

In our simulations, a coherent excitation, given by the breathing mode oscillation of the beam envelope, interacts with a large set of oscillators, the beam particles, oscillating with a large betatron frequency spectrum. The space charge force, which induces the tune shift in the particle betatron oscillation, is time varying because the beam is oscillating in breathing mode. This results in a parametric coupling between the coherent and the betatron oscillations. Parametric resonances occur when the time varying parameter (in our case, the space charge term) oscillates at twice the fundamental frequency (in our case, betatron frequency) [12]. For this reason, in order to obtain a resonant transfer of energy from the coherent oscillation to the incoherent oscillations, half of the coherent frequency must lay inside the betatron frequency spectrum. The rms envelope oscillations shown in Fig. 1 have frequencies of $\omega^{\mathrm{o}}=76 \mathrm{MHz}$ for the odd mode and $\omega^{\mathrm{e}}=90 \mathrm{MHz}$ for the even mode. From Fig. 2(a) one can notice that the odd mode half frequency stays inside the betatron spectrum, while the even mode is outside. Being satisfied of the existence of the required conditions, the Landau damping mechanism occurs. This conclusion seems to be confirmed by the results shown in Fig. 1(a) for the odd mode. However, a more accurate interpretation of the results shown in Fig. 1 requires further consideration. In fact, in Fig. 1(a) we can observe that the fast damping starts after about 140 focusing periodic cells. This is because the initial K-V distribution of the beam changes, as observed previously, just in about $140 N p[2,4]$. During this rearranging process the beam emittance increases as shown in Fig. 1(b). A further slight emittance increase can be noticed after this process is finished. Precisely, it occurs during the fast damping of the beam breathing mode oscillation. This effect can be ascribed to the energy transfer from the coherent oscillation towards the incoherent oscillations of the beam particles. In other words, the energy involved in this process is dissipated from a coherent motion towards the very high number of degrees of freedom of the system.

For the even mismatch case (magenta line), the half frequency has a value of $45 \mathrm{MHz}$ and it is practically outside or at least on the edge of the betatron frequency spectrum [see Fig. 2(a)]. Following the previous arguments, only a very slight Landau damping could occur in this case and the results, shown in Fig. 1(a), confirm this prediction. The fast damping begins immediately when an initial Gaussian distribution is used (see Fig. 3). In this case, a large betatron spectrum characterizes the beam from the beginning. Its frequency distribution is shown in Fig. 4. This time the Landau damping conditions are matched from the onset of the breathing oscillation modes. However, the partial damping observed in Fig. 3, for the even mode placed on the edge of the frequency distribution, could be due to the rearrangement of the particle charges from the Gaussian towards the equilibrium distribution (flat with Debye length tails [10]). This redistribution produces a shrinkage of the original betatron spectrum.

In the simulation results shown in Fig. 5, a value of $\sigma / \sigma_{0}=0.83$ (corresponding to $I=24 \mathrm{~mA}$ ) was considered. In this case, the beam betatron spectrum, shown in Fig. 2(b), is a Dirac delta so that the Landau damping condition of a large betatron spectrum is not satisfied. Therefore, the envelope oscillation amplitudes for the above case 
do not present fast damping (Fig. 5). In Figs. 6 and 7, for the same value of $\sigma / \sigma_{0}=0.83$, an initial beam distribution of Gaussian type is considered. The related frequency distribution is shown in Fig. 6. In this case, the Landau damping conditions are again matched. It can be seen from Fig. 7 that both the even and the odd oscillation modes undergo the fast damping. This is because the half frequencies, $\omega^{\mathrm{o}} / 2=50$ and $\omega^{\mathrm{e}} / 2=53 \mathrm{MHz}$, lay inside the betatron spectrum, thus leading to a quick damping for both modes. However, it must be noticed that the odd mode damps slightly faster than the even mode. The reason can be inferred from the relation (1), where it can be

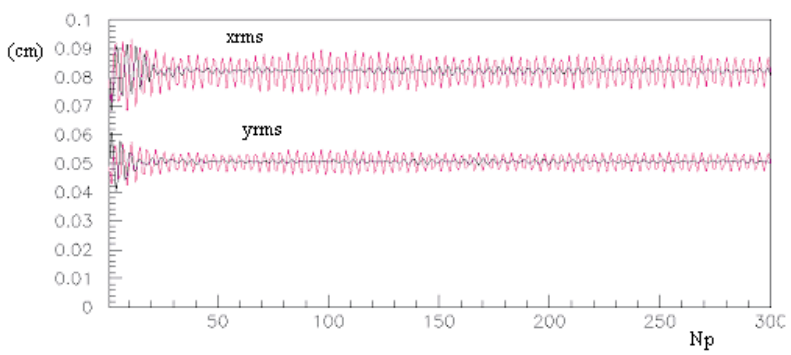

FIG. 3. (Color) The rms envelope oscillations with initial Gaussian distribution for $\sigma / \sigma_{0}=0.55$. The black line refers to the odd mode and the magenta line to the even mode.

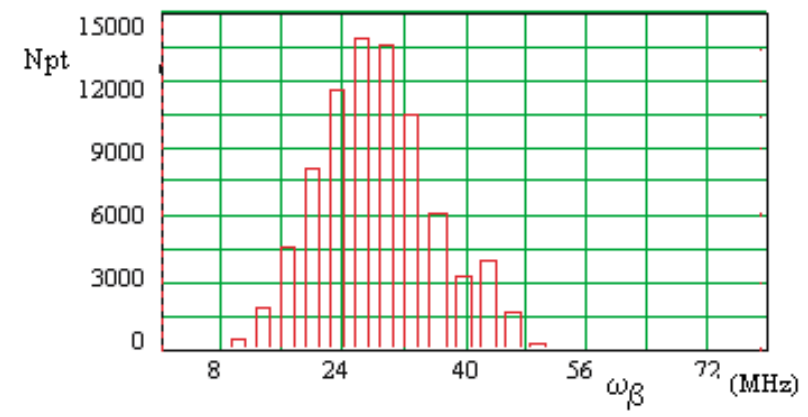

FIG. 4. (Color) Beam betatron frequency distribution with initial Gaussian distribution for $\sigma / \sigma_{0}=0.55(I=95 \mathrm{~mA})$. The odd mode half frequency, $\omega^{\circ}$, is $38 \mathrm{MHz}$ and the even mode half frequency, $\omega^{\mathrm{e}}$, is $45 \mathrm{MHz}$.

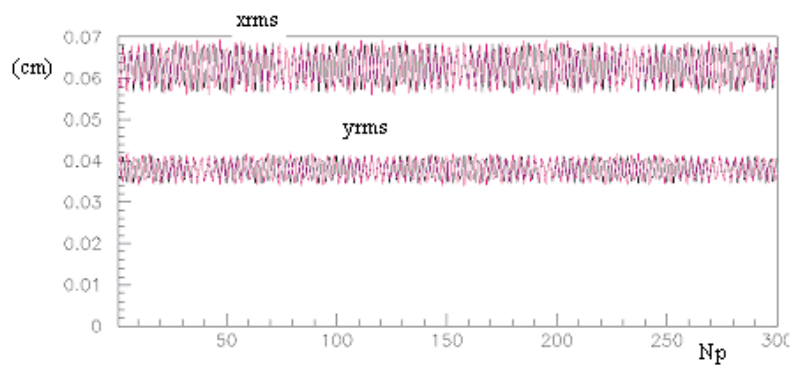

FIG. 5. (Color) The rms envelope oscillations with initial K-V distribution for $\sigma / \sigma_{0}=0.83$. The black line refers to the odd mode and the magenta line to the even mode. seen that the damping strength is proportional to $f(\omega)$, the betatron spectrum density for $\omega$.

In order to collect more evidence on the role of Landau mechanisms of stabilization in the origin of the fast damping observed, further simulations are considered for different values of $\sigma / \sigma_{0}$ with K-V and Gaussian initial beam distributions.

Figure 8 shows the simulation results for $\sigma / \sigma_{0}=0.29$ (corresponding to a beam current $I=200 \mathrm{~mA}$ ), with an

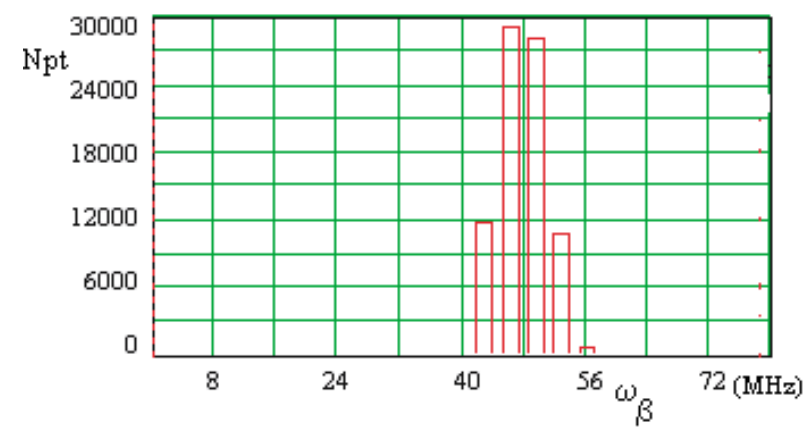

FIG. 6. (Color) Beam betatron spectrum with initial Gaussian distribution for the case $\sigma / \sigma_{0}=0.83$ (corresponding to $I=$ $24 \mathrm{~mA}$ ). The odd mode half frequency, $\omega^{\circ}$, is $50 \mathrm{MHz}$ and the even mode half frequency, $\omega^{\mathrm{e}}$, is $53 \mathrm{MHz} . N p t$ is the particle number.

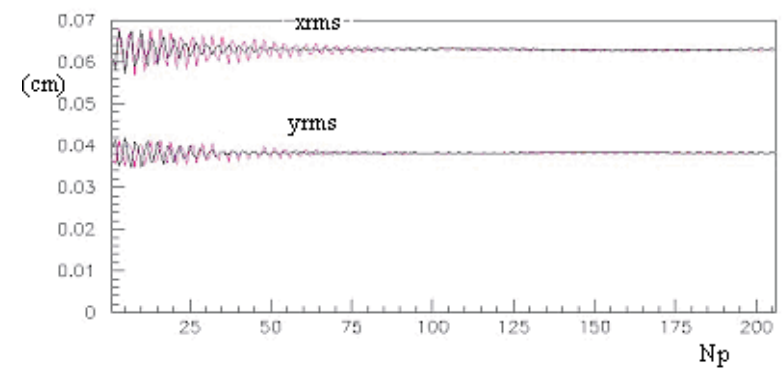

FIG. 7. (Color) The rms envelope oscillation with Gaussian distribution for the case $\sigma / \sigma_{0}=0.83$. The black line represents the odd mode and the magenta line represents the even mode.

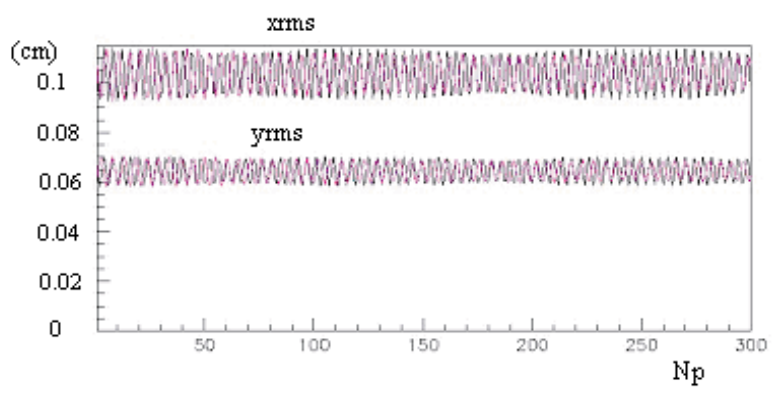

FIG. 8. (Color) The rms envelope oscillation damping with initial K-V distribution for $\sigma / \sigma_{0}=0.29(I=200 \mathrm{~mA})$. The black line refers to the odd mode and the magenta line to the even mode. 


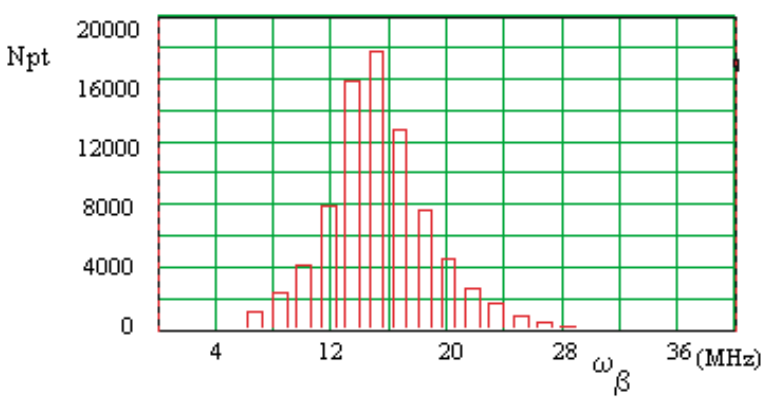

FIG. 9. (Color) Beam betatron frequency distribution with initial $\mathrm{K}-\mathrm{V}$ particle distribution for $\sigma / \sigma_{0}=0.29$. The odd mode half frequency, $\omega^{\circ}$, is $32 \mathrm{MHz}$ and the even mode half frequency, $\omega^{\mathrm{e}}$, is $42 \mathrm{MHz}$. They are both outside of the spectrum.

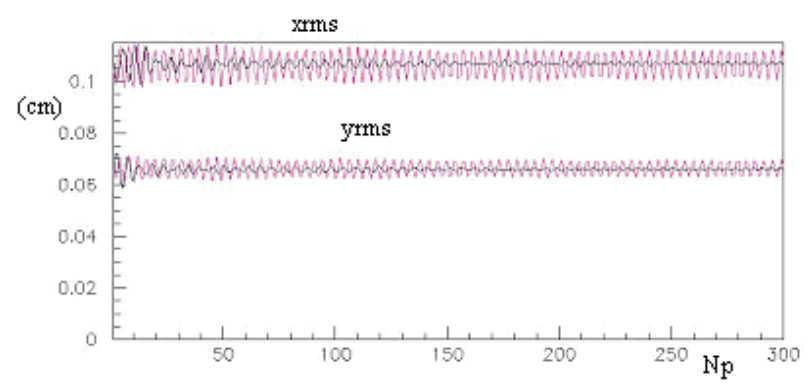

FIG. 10. (Color) The rms envelope oscillation damping with initial Gaussian particle distribution for $\sigma / \sigma_{0}=0.29(\infty=$ $200 \mathrm{~mA}$ ). The black line refers to the odd mode and the magenta line to the even mode.

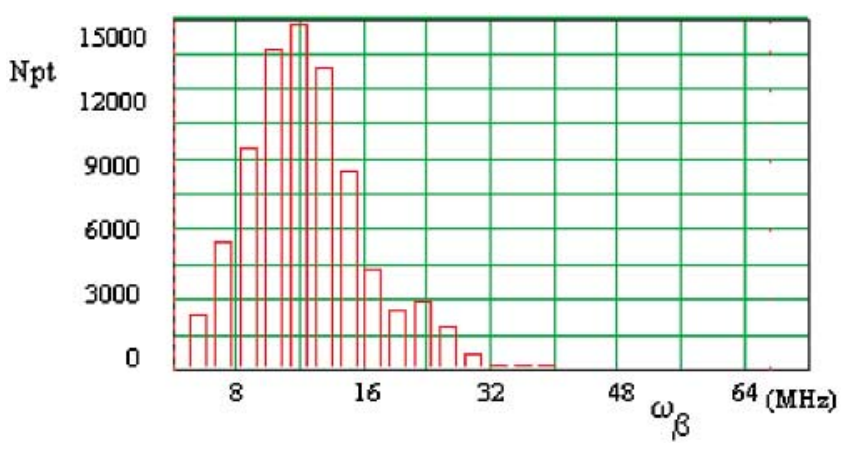

FIG. 11. (Color) Beam betatron frequency distribution with initial Gaussian particle distribution for $\sigma / \sigma_{0}=0.29$ $(I=200 \mathrm{~mA})$. The odd mode half frequency, $\omega^{\mathrm{o}}$, is $32 \mathrm{MHz}$ and the even mode half frequency, $\omega^{\mathrm{e}}$, is $42 \mathrm{MHz}$.

initial beam particle distribution of $\mathrm{K}-\mathrm{V}$ type. In this case, the rms envelope oscillation modes have $\omega^{\circ} / 2=32$ and $\omega^{\mathrm{e}} / 2=42 \mathrm{MHz}$. Both of these frequencies are outside of the corresponding betatron spectrum presented in Fig. 9. According to the previous considerations, no damping can be observed on either of the rms envelop oscillation modes of Fig. 8.
The simulations for the same case with an initial distribution of Gaussian type are shown in Fig. 10. This time the betatron spectrum is larger, so that the odd mode half frequency falls inside of it, as can be seen from Fig. 11. Then, as argued before, only the odd mode will undergo a fast damping, as confirmed by the results shown in Fig. 10.

\section{CONCLUSIONS}

In conclusion, the simulation results confirm that the damping effect shown in some cases is a consequence of the Landau mechanism of stabilization. Through this mechanism, a coherent oscillation (the envelope breathing mode) transfers its energy to the many degrees of freedom of an ensemble of oscillating systems (the beam particles oscillating at the betatron frequencies). Furthermore, a fast damping of the mismatched envelope oscillations in space charge dominated beams (see the case $\left.\sigma / \sigma_{0}=0.55\right)$ is observed also for initial K-V distributions. This, however, occurs only after a tail is formed on the initial $\mathrm{K}-\mathrm{V}$ distribution, due to internal rearrangement of the beam particle charges [10]. This process enlarges the beam betatron spectrum allowing a matching of the Landau damping conditions. On the other hand, when the abovementioned Landau damping conditions are satisfied with an initial Gaussian distribution, the simulation results show a fast damping starting from the onset of the transportation through the periodic cells. Although the simulations shown in this paper give, in my opinion, strong evidence that the fast damping effect is caused by a Landau damping mechanism of stabilization, for a complete demonstration a detailed analytical discussion is needed and is under way.

[1] H. Koziol, Los Alamos MP Division Report No. MP3-75-1, 1975.

[2] T.P. Wangler, K. R. Crandall, R. S. Mills, and M. Reiser, IEEE Trans. Nucl. Sci. 32, 2196 (1985).

[3] M. Reiser, in Proceedings of the IEEE Particle Accelerator Conference, San Francisco, 1991, edited by L. Lizama and J. Chew (IEEE, New York, 1991), p. 2497.

[4] V. Variale, Nuovo Cimento Soc. Ital. Fis. 112A, 1571-1582 (1999).

[5] T. Clauser et al., in Proceedings of the Particle Accelerator Conference, New York, 1999 (IEEE, Piscataway, NJ, 1999), p. 1779.

[6] A. Hoffmann, Report No. CAS CERN 95-06, Vol. I, 1995.

[7] J. Struckmeier, GSI-Darmstadt Report No. GSI-ESR-03.

[8] J. L. Laclare, Report No. CAS CERN85-19, Vol. II, 1985.

[9] T.P. Wangler, in Space Charge Dominated Beams \& Applications of High Brightness Beams, edited by S. Y. Lee, AIP Conf. Proc. No. 377 (AIP, New York, 1994).

[10] I. Hofmann, Applied Charged Particle Optics, edited by A. Septier (Academic, New York, 1983).

[11] J. Struckmeier and M. Reiser, Part. Accel. 14, 227 (1984).

[12] W. H. Louisell, Coupled Mode and Parametric Electronics (Wiley, New York, 1960). 